05.3

\title{
Влияние кинетики роста новой фазы и давления на распределение компонентов при фазовых переходах
}

\author{
() А.П. Гуськов \\ Институт фризики твердого тела РАН, Черноголовка, Московская обл., Россия \\ E-mail: guskov@issp.ac.ru
}

Поступило в Редакцию 12 ноября 2020г.

В окончательной редакции 15 февраля 2021 г.

Принято к публикации 1 марта 2021г.

\begin{abstract}
Показана взаимосвязь между кинетикой присоединения частиц раствора к новой фазе, изменением количества движения компонента на межфазной границе и диффузией компонентов в фазах. Это взаимодействие существенно влияет на распределение концентрации компонентов в фазах. Предложенная теория объясняет зависимость эффективного коэффициента распределения от концентрации при зонной очистке растворов.
\end{abstract}

Ключевые слова: фазовый переход, раствор, диффузия, неравновесная термодинамика.

DOI: 10.21883/PJTF.2021.11.50999.18613

Известное распределение компонентов раствора при фазовых переходах не учитывает кинетику присоединения частиц компонентов к новой фазе [1]. Цель настоящей работы - показать, что кинетика присоединения частиц компонентов к новой фазе кардинально влияет на распределение их концентрации в фазах. Рассмотрим фазовый переход двухкомпонентного раствора. Межфазную границу считаем плоской и резкой, так же как при постановке квазиравновесной задачи диффузии в работе [1]. Запишем обобщенный закон Фика [2] для компонента $B$ фазы $i$ в координатах, неподвижных относительно межфазной границы $(w-$ скорость раствора). Градиент температуры считаем малым. Вместе с уравнением неразрывности получаем систему уравнений относительно концентрации и парциальной скорости компонента $w_{B i}(z)$

$$
\begin{gathered}
c_{B i}(z)\left(w_{B i}(z)-w\right)=-D_{i} \frac{d c_{B i}(z)}{d z}-\vartheta_{L B i} \frac{d p(z)}{d z}, \\
\frac{d}{d z}\left(c_{B i}(z) w_{B i}(z)\right)=0 .
\end{gathered}
$$

Здесь $c_{B i}(z)$ - распределение концентрации компонента $B, p(z)$ - давление, $z$ - пространственная переменная, $D_{i}-$ коэффициент диффузии, $\vartheta_{L B i}-$ парциальный удельный (мольный) объем, $w_{B i}(z)$ - парциальная гидродинамическая скорость компонента $B_{i}, i$ - твердая или жидкая фаза (sol или liq соответственно). Формально неизвестная функция $p(z)$ требует уравнения сохранения количества движения. Однако упрощенная постановка задачи и условие одномерности позволяют найти давление, связанное с изменением концентрации раствора при его переходе через межфазную границу. Слагаемое с градиентом давления имеет простой физический смысл: это сила, которая действует на частицы компонентов раствора из-за изменения их парциальных скоростей. Внешние силы, перемещающие раствор, в уравнение Фика не входят. Они должны войти в уравнение движения. Схема введения этих сил зависит от причин, которые вызывают перемещение раствора. Вместо внешних сил в задаче задается скорость перемещения межфазной границы. Перемещение раствора и компонентов предполагается ламинарным. Преобразованием системы уравнений (1) нетрудно получить уравнение

$$
D_{i} \frac{\partial^{2} c_{B i}(z)}{\partial z^{2}}-\frac{1}{D_{i}}\left(w c_{B i}(z)-j_{B i}\right) w=0,
$$

где $n=A, B, j_{n i}=c_{n i}(z) w_{n i}(z)=$ const, так как в любом сечении системы массовый поток любого компонента не зависит от координаты. Решением уравнения (2) является функция

$$
c_{B i}(z)=A_{1 B i} \exp \left(\frac{w z}{D_{i}}\right)+A_{2 B i} \exp \left(-\frac{w z}{D_{i}}\right)+\frac{j_{B i}}{w} .
$$

При $\vartheta_{L B i}=0$ преобразованием системы уравнений (1) также можно получить уравнение (2). Но в этом случае решение (3) не удовлетворяет уравнению Фика (1). По этой причине известная квазиравновесная модель распределения концентрации при кристаллизации [1] дает постоянную концентрацию в одной из фаз. Решение в твердой фазе рассматривается на полуограниченном интервале, поэтому $A_{1 B s o l}=0$. На межфазной границе запишем равенство $c_{\text {Bsol }}(0) w_{B s o l}(0)=c_{B l i q}(0) w_{B l i q}(0)$. Подставляя соответствующие выражения из закона Фика, приходим к уравнению

$$
2 w\left(A_{2 B s o l}-A_{2 B l i q}\right)+j_{B s o l}-j_{B l i q}=0 .
$$

При условии сохранения массовых потоков компонентов $j_{B s o l}-j_{\text {Bliq }}=0$ это условие выражает сохранение количества движения компонента $B$ на межфазной границе. Пусть жидкая фаза ограничена координатой $z_{0}$ с заданной концентрацией $c_{B l i q}\left(z_{0}\right)$. Из уравнений (4), $c_{B l i q}\left(z_{0}\right)=C_{i n i}$ и условия сохранения массового потока 
находятся $A_{2 \text { Bliq }}, A_{2 B s o l}$ и $A_{1 \text { Bliq. }}$. При $\frac{w z}{D_{l i q}} \ll 1$ решения существенно упрощаются и принимают вид

$$
\begin{gathered}
c_{\text {Bsol }}(z)=a c_{\text {Bliq }}\left(z_{0}\right) D_{l i q}\left(\left(k_{e} w-w_{\text {Bliq }}(0)\right)\right. \\
\left.\times \exp \left(-\frac{w z}{D_{s o l}}\right)+w_{B l i q}(0)\right), \\
c_{B l i q}(z)=a c_{B l i q}\left(z_{0}\right) D_{l i q}\left(w\left(1-k_{e}\right) \exp \left(\frac{w z}{D_{l i q}}\right)\right. \\
\left.+\left(k_{e} w-w_{\text {Bliq }}(0)\right) \exp \left(-\frac{w z}{D_{l i q}}\right)+w_{\text {Bliq }}(0)\right),
\end{gathered}
$$

где $a=w\left(\left(w_{\text {Bliq }}(0)-2 k_{e} w+w\right) z_{0}+D_{l i q}\right), k_{e}-$ равновесный коэффициент сегрегации (линеаризованная равновесная фазовая диаграмма). Зная распределение концентрации, из закона Фика (1) получаем распределение давления

$$
\vartheta_{L B i} p(z)=\int\left(c_{B i}(z) w-D_{i} \frac{\partial c_{B i}(z)}{\partial z}-j_{B i}\right) d z+A_{p B i}
$$

Здесь постоянная интегрирования $A_{p B i}$ не зависит от $z$.

В виде (5), (6) решение не дает предельного перехода к равновесию. Если подставить сюда $w=0$, получим расходимость концентрации в обеих фазах. Этот эффект объясняется тем, что решение не учитывает отклонение системы от равновесия. Для каждого компонента существует зависимость парциальной скорости от разности между равновесной температурой фазового перехода и температурой межфазной границы $\Delta T_{k}=T_{e}-T(0)$ (так называемое кинетическое переохлаждение). Введем кинетические зависимости для скорости $w_{\text {Bliq }}(z)$ и скорости раствора $w$. Такой выбор скоростей удобен для того, чтобы показать связь полученных решений с известными квазиравновесными решениями [1]. Если учесть, что скорости входят в полные решения в виде отношения $w / w_{\text {Bliq }}(z)$, нетрудно видеть, что кинетические зависимости имеют следующие свойства.

1. Разложение в ряд Маклорена функций $w_{l i q}(0)$ и $w$ по кинетическому переохлаждению $\Delta T_{k}$ не содержит слагаемого 0-го порядка.

2. Слагаемые с наименьшими степенями этих разложений имеют одинаковые степени.

3. Коэффициенты при наименьших степенях $\Delta T_{k}$ равны.

Простейшими зависимостями $w_{B l i q}(0)$ и $w$, которые удовлетворяют этим трем условиям, являются функции

$$
w=k_{e} h \Delta T_{k}+\left(1-k_{e}\right) h_{B} \Delta T_{k}^{2}, \quad w_{B l i q}(0)=k_{e} h \Delta T_{k},
$$

где $h$ и $h_{B}$ - кинетические коэффициенты. В случае азеотропных растворов (фазы с равными составами), $k_{e} \rightarrow 1$, получаем $w_{\text {Bliq }}(0) \rightarrow w$. При $k_{e} \rightarrow 0$, т.е. малой растворимости компонентов в твердой фазе, $w_{\text {Bliq }}(0) \rightarrow 0$. В этом случае необходимо учитывать подвижность компонента $B$. В результате подстановки (8)
Значения параметров, используемых в численных расчетах

\begin{tabular}{c|c|c|c|c|c|c|c|c}
\hline$k_{e}$ & $h$ & $h_{B}$ & $C_{i n i}$ & $\vartheta_{\text {LBliq }}$ & $\vartheta_{\text {LBsol }}$ & $P_{\text {ext }}$ & $D_{\text {liq }}$ & $D_{\text {sol }}$ \\
\hline 0.6 & -0.1 & -2.8 & 0.15 & -4.5 & 0.1 & 1 & 1 & 0.1
\end{tabular}

в решения задачи получим зависимости распределения концентрации и давления от $z$ и $\Delta T_{k}$.

Чтобы сравнить полученные расчеты с решением известной квазиравновесной задачи диффузии [1], предположим, что кристаллизуется жидкий раствор. Исходные параметры представлены в таблице. Значения параметров расчетов берутся без привязки к размерностям, чтобы на графиках было удобно интерпретировать физическую связь между скоростями, концентрацией и давлением. Зависимости концентрации от пространственной координаты и кинетического переохлаждения представлены на рис. 1 . Постоянные концентрации $1 L$ и $1 S$ соответствуют равновесию $\Delta T_{k}=0$. Из общих решений и решений $(5),(6)$ следует, что при $w_{B l i q}(0)=k_{e} w$ концентрация в твердой фазе и давление в обеих фазах постоянные (кривые $3 S$ на рис. 1,2 и $3 L$ на рис. 2). Обозначим соответствующее значение кинетического переохлаждения $\Delta T_{k}=\Delta T_{P}$. Из рис. 1 и 2 легко видеть связь между распределением концентрации и давлением. При $\Delta T_{k}<\Delta T_{P}$ концентрация в твердой фазе убывает (кривая $2 S$ ), а при $\Delta T_{k}>\Delta T_{P}$ (кривые $4 S-6 S$ ) возрастает от $z=-\infty$ до межфазной границы. Кривая $3 S$ является граничной между областями возрастания и убывания концентрации. Причина такого поведения состоит в том, что в твердой фазе при $\Delta T_{k}<\Delta T_{P}$ давле-

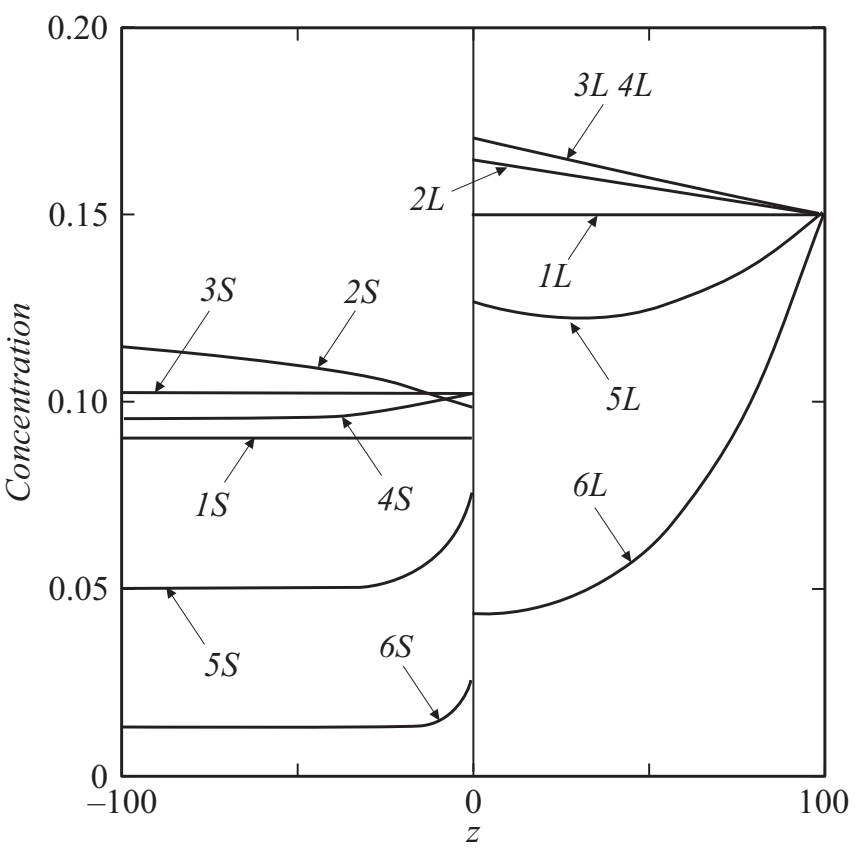

Рис. 1. Распределение концентрации в окрестности межфазной границы. $S-$ твердая фаза, $L-$ жидкая фаза; $\Delta T_{k}=0(1)$, $0.021(2), 0.035\left(\Delta T_{k}=h / h_{b}\right)(3), 0.042(4), 0.080(5), 0.124$ (6). 


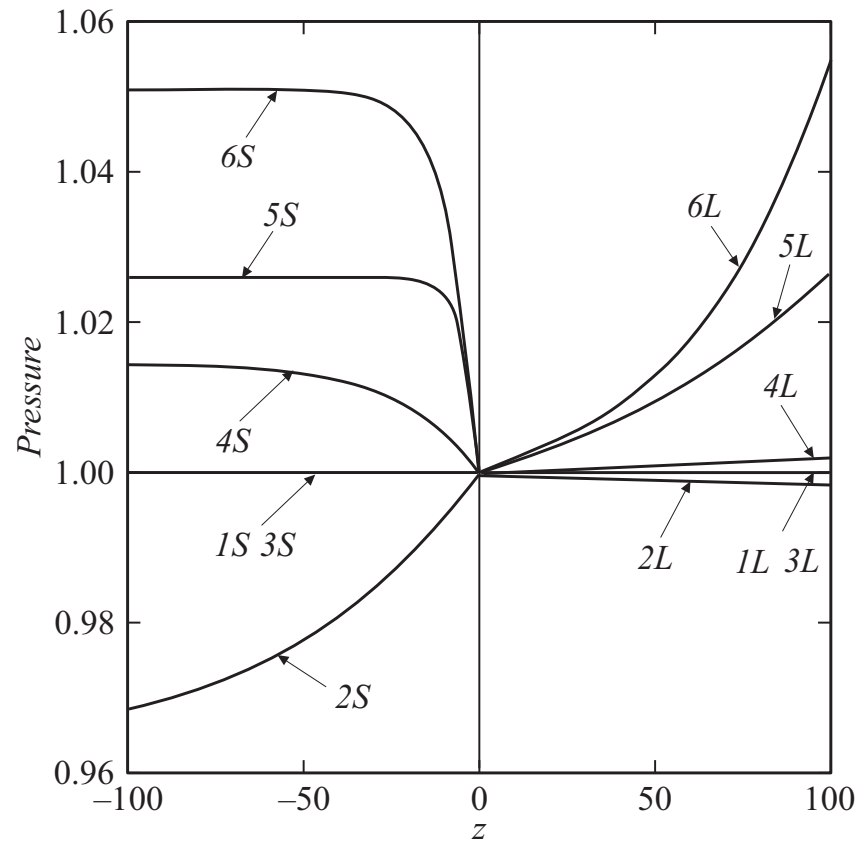

Рис. 2. Распределение давления в окрестности межфазной границы. $S$ - твердая фаза, $L-$ жидкая фаза; $\Delta T_{k}=0(1)$, 0.021 (2), $0.035\left(\Delta T_{k}=h / h_{b}\right)(3), 0.042$ (4), 0.080 (5), 0.124 (6).

ние возрастает (кривая $2 S$ на рис. 2), а при $\Delta T_{k}>\Delta T_{P}$ (кривые $4 S-6 S$ на рис. 2) убывает. Следовательно, сила, действующая на компонент $B$, при $\Delta T_{k}=\Delta T_{P}$ изменяет направление. В жидкой фазе при постоянном давлении (кривая $3 L$ на рис. 2) концентрация не постоянная (кривая $3 L$ на рис. 1). Этому решению соответствует решение квазиравновесной задачи [1]. Других решений квазиравновесная задача не дает, потому что она не учитывает давление, связанное с процессом диффузии компонентов раствора.

Квазиравновесное решение используется, например, в теории зонной очистки растворов Бартона-Прима-Слихтера (BPS) при фазовых переходах [1]. Для расчетов степени очистки в теории BPS вводится эффективный коэффициент сегрегации $k_{e f f}$. Он равен отношению $c_{B s o l}(0) / c_{B l i q}\left(z_{0}\right)$. В работе [3] был проведен анализ влияния условий эксперимента на значения $k_{e f f}$. Значения $k_{\text {eff }}$ рассчитывались по известным методикам с использованием экспериментальных данных. В частности, был проведен анализ влияния числа проходов зонной плавки на величину $k_{e f f}$ при постоянной скорости ее перемещения. В результате было показано, что $k_{e f f}$ существенно зависит от числа проходов. При каждом проходе зоны плавления изменяется количество компонента примеси в растворе. Следовательно, $k_{\text {eff }}$ зависит от значения концентрации $c_{B l i q}\left(z_{0}\right)$. Теория BPS дает зависимость $k_{e f f}$ только от $k_{e}, w$ и $D_{l i q}$. Объясним полученную в экспериментах зависимость. Легко видеть, что отношение $c_{B s o l}(0) / c_{\text {Bliq }}\left(z_{0}\right)$ при распределении концентрации (5), (6) зависит также от парциальной ско- рости $w_{\text {Bliq }}(0)$. Скорость $w_{\text {Bliq }}(0)$ связана со скоростью раствора соотношением

$$
w=c_{A i}(z) w_{A i}(z)+c_{B i}(z) w_{B i}(z) .
$$

На межфазной границе сюда войдет величина $c_{B l i q}(0)$, которая, согласно (6), зависит от граничной концентрации $c_{B l i q}\left(z_{0}\right)$. Следовательно, рассматриваемая задача дает зависимость эффективного коэффициента распределения от $c_{B l i q}\left(z_{0}\right)$. Следует подчеркнуть, что зависимость $k_{e f f}\left(c_{B l i q}\left(z_{0}\right)\right)$ дает кинетика присоединения частиц компонентов раствора к новой фазе, т.е. она связана с отклонением системы от равновесия. Численный расчет по упрощенному варианту соотношений кинетики (8) не даст такой зависимости, так как в нем скорость раствора $w$ напрямую связана с кинетическим переохлаждением. Если положить $w_{\text {Aliq }}(0)=h_{a} \Delta T_{k}$ и $w_{B l i q}(0)=h_{b} \Delta T_{k}$, то при постоянных параметрах $h_{a}, h_{b}$ выражение (9) дает зависимость скорости раствора от $c_{\text {Bliq }}\left(z_{0}\right)$. На рис. 3 показана зависимость $k_{e f f}$ от граничной концентрации $c_{B l i q}\left(z_{0}\right)$ при значениях $w=10^{-5}$, $h_{a}=10^{-5}, h_{b}=10^{-6}, D_{l i q}=10^{-8}, z_{0}=0.001$. Как и в работе [3], $k_{e f f}$ убывает при уменьшении $c_{B l i q}\left(z_{0}\right)$.

Таким образом, можно сделать следующие выводы.

1. При фазовых переходах происходит взаимодействие между кинетикой присоединения частиц раствора к новой фазе, давлением и диффузией компонентов в фазах.

2. Обобщенный закон Фика дает условие сохранения количества движения компонента на межфазной границе.

3. При описании стационарного процесса распределения компонентов в процессе фазового перехода раствора необходимо учитывать степень отклонения фаз раствора от равновесия.

4. Эффективный коэффициент распределения зависит от концентрации компонентов в зоне плавления при зонной очистке растворов.

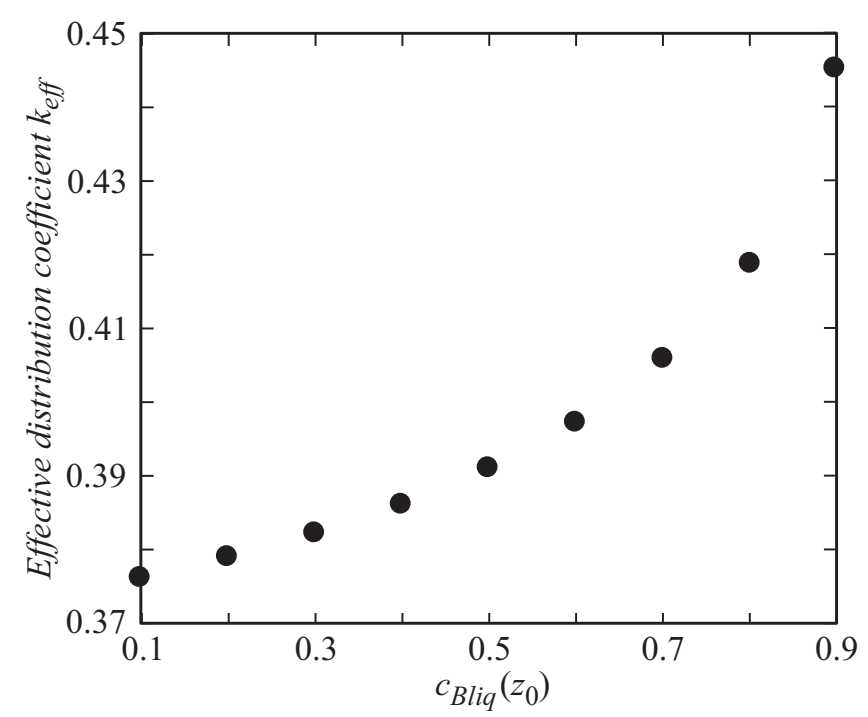

Рис. 3. Зависимость эффективного коэффициента распределения от граничной концентрации. 


\section{Конфликт интересов}

Автор заявляет, что у него нет конфликта интересов.

\section{Список литературы}

[1] K.A. Jackson, Kinetic processes: crystal growth, diffusion, and phase transitions in materials (Willey-VCH Verlag, Weinheim, 2004). DOI: 10.1002/3527603891

[2] И. Пригожин, Д. Кондепуди, Современная термодинамика (Мир, М., 2002).

[3] H. Wan, J. Zha, B. Yang, B. Xu, M. Duan, L. Kong, Y. Dai, J. Mater. Res. Technol., 9 (5), 10366 (2020). https://doi.org/10.1016/j.jmrt.2020.07.050 